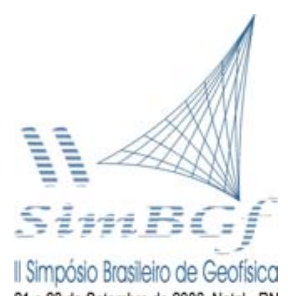

21 a 23 de Setembro de 2006 , Natal - RN

\title{
Ambiente Gráfico Para Imageamento Sísmico
}

Jeferson A. Correia de Andrade e Reynam da C. Pestana, UFBA - CPGG Salvador, Bahia, Brasil.

Copyright 2006, SBGf - Sociedade Brasileira de Geofísica

Este texto foi preparado para a apresentação no II Simpósio de Geofísica da Sociedade Brasileira de Geofísica, Natal/RN, 21-23 de setembro de 2006. Seu conteúdo foi revisado pela Comissão Tecno-científica do II SR-SBGf mas não necessariamente representa a opinião da SBGf ou de seus associados. E proibida a reprodução total ou parcial deste material para propósitos comerciais sem prévia autorização da SBGf.

\section{Resumo}

Este trabalho tem como principal objetivo apresentar um ambiente gráfico (GUI) que foi desenvolvido e implementado para os métodos de migração de dados sísmicos desenvolvidos no LAGEP/CPGG/UFBA. A partir dessa nova interface gráfica os algoritmos de imageamento sísmico podem ser agora utilizados de forma mais amigável e também novos métodos de migração podem ser facilmente testados e incorporados a esse novo sistema. Assim, os alunos e os pesquisadores do LAGEP passarão a dispor de um ambiente gráfico de imageamento sísmico bastante eficiente e de fácil manuseio. O sistema apresentado aqui foi testado com sucesso em dois conjuntos de dados sísmicos sintéticos.

\section{Introdução}

A identificação de estruturas, possivelmente portadoras de hidrocarbonetos, em áreas de alta complexidade estrutural é prejudicada pela baixa qualidade da imagem sísmica obtida após o processamento dos dados sísmicos, o que torna arriscada a interpretação dos dados e a avaliação de prospectos em tal situação geológica. Os métodos de imageamento de dados sísmicos mais adequados para áreas de alta complexidade estrutural, associada a fortes variações laterais de velocidades, são os métodos de migração préempilhamento em profundidade. A migração em profundidade é baseada na solução da equação acústica da onda, como exemplo, temos a migração Kirchhoff e as técnicas de migração espectral, tais como: Phase-Shift, Phase-Shift mais interpolação (PSPI) e migração em duas etapas (Split-Step). A construção do campo de velocidades em profundidade também pode ser feita com técnicas de análise de velocidade por migração e, assim, obtendo-se um campo de velocidades em profundidade com uma melhor precisão. A utilização de técnicas de migração pré-empilhamento em profundidade, a construção do campo de velocidades, obtido através da integração do conhecimento geológico e técnicas de análise de velocidade por migração, são as ferramentas geofísicas mais adequadas para o tratamento de dados sísmicos provenientes de situações geológicas complexas. E conseqüentemente a aplicação de técnicas de imageamento sísmico irá certamente melhorar a identificação de estruturas, possivelmente com hidrocarbonetos, possibilitando uma diminuição substancial dos riscos na localização de prospectos de exploração geofísica. De uma forma geral, o desenvolvimento das técnicas de imageamento destinadas à melhoria da identificação de estruturas situadas em áreas de alta complexidade estrutural, ajudará na interpretação sísmica e, assim, uma conseqüente redução do risco exploratório.

O ambiente gráfico foi concebido e desenvolvido com o intuito de possibilitar a utilização o mais amigável possível dos métodos de migração sísmica desenvolvidos no CPGG/UFBA. Então interfaces gráficas foram criadas usando a biblioteca Qt da Trolltech, que tem como linguagem de estrutura a linguagem $\mathrm{C}++$ criada por Bjarne Stroustrup. E a partir da criação desse novo ambiente gráfico proporcionaremos aos alunos e pesquisadores do CPGG/UFBA uma utilização mais simples e bastante eficiente dos métodos de imageamento sísmicos existentes nesta Instituição. Além disso, a criação desse sistema gráfico permitirá ainda a incorporação de novos algoritmos de migração, bem com a realização de testes e também a execução de novos programas de uma forma bastante rápida e amigável.

\section{Metodologia/ Problema Investigado}

Este trabalho foi realizado no Laboratório de Geofísica de Exploração de Petróleo (LAGEP) no CPGG/UFBA e teve apoio do convênio da ANP/UFBA, da pós-graduação em Geofísica e do projeto 01/02 da Rede de Risco Exploratório - CTPETRO/CNPq - FINEP. Para o desenvolvimento das etapas visando a implementação de um ambiente gráfico, para o sistema de imageamento sísmico, utilizamos a infra-estrutura computacional instalada no LAGEP. Os equipamentos utilizados por este trabalho de pesquisa foram os seguintes:

Rede de computadores (sistema operacional Linux); INFOCLUSTER ITAUTEC ${ }^{\circledR}$ de 21 nós, com processadores Intel ${ }^{\circledR}$ Xeon ${ }^{\circledR}$ duais de $2.6 \mathrm{GHz}, 2 \mathrm{~GB}$ de memória RAM e 40 GB de disco; Impressora Post-Script Laser.

Nossas atividades foram, principalmente, realizadas em um computador com processador Intel $\AA$ Pentium ${ }^{\circledR} 4$ de $2.4 \mathrm{GHz}$ e $2 \mathrm{~GB}$ de memória RAM, com os seguintes sistemas instalados:

Red Hat Linux® v. 9.0 (sistema operacional); Software Qt Designer; g++ (compilador $\mathrm{C}++$ ); qmake (compilador Qt); Pacote de processamento Sísmico Seismic Unix da Colorado School of Mines. 
Para criar o nosso ambiente gráfico de imageamento de dados sísmicos iniciamos fazendo uma pesquisa para identificar um programa de domínio público, que nos permitisse o desenvolvimento de interfaces gráficas. Então, optamos pelo software Qt da Trolltech, pois o mesmo possui uma vasta biblioteca gráficas e também uma boa documentação "on-line".

\section{Resultados}

Com a utilização da linguagem de programação $\mathrm{C}++$, associado ao pacote de bibliotecas Qt da Trolltech, foi possível desenvolver um ambiente gráfico (Figura. 1) para a utilização de métodos de imageamento sísmico desenvolvidos no CPGG/UFBA. Esta interface gráfica apresenta uma estrutura leve com textos explicativos e de fácil entendimento. Ela possibilitará a implementação de novos métodos e testes de algoritmos de uma forma mais eficiente, pois possui uma estrutura bastante simples e de fácil manuseio. A interface gráfica está disponível aos alunos e pesquisadores de Geofísica da UFBA e também está instalado no "Cluster" de PC's INFOCLUSTER ITAUTEC do Laboratório de Geofísica de Exploração de Petróleo - LAGEP do CPGG/UFBA.

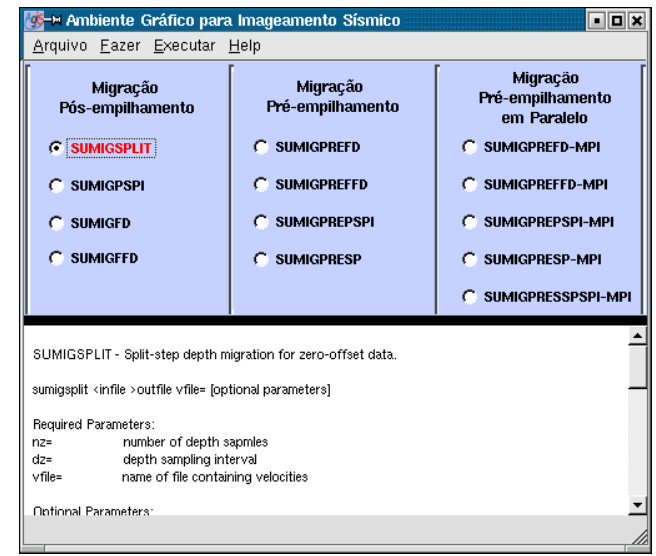

Figura 1 - Ambiente Gráfico para Imageamento Sísmico - AGIS. Tela principal.

Aplicamos duas técnicas de migração aos dados sísmicos, um conjunto de dados pré-empilhados ordenados em famílias de ponto de tiro comum, gerado a partir do modelo Marmousi e outro pós-empilhado gerado a partir do domo de sal SEG-EAGE para teste deste novo programa (Figura1) desenvolvido no LAGEP.

A primeira técnica de migração aplicada foi a migração "Split-Step" pós-empilhamento em profundidade usando o dado sintético domo de sal SEG-EAGE (Figura 3). A segunda técnica aplicada foi a migração "Split-Step" préempilhamento em profundidade usando o dado sintético Marmousi (Figura 5).

O dado sintético domo de sal SEG-EAGE simula uma estrutura geológica de sal com forte contraste de velocidade baseado numa estrutura existente no Golfo do México e consiste de 325 famílias de tiros com intervalo de amostragem de $8 \mathrm{~ms}$ e com 626 amostras por traço. Seu campo de velocidade é mostrado na Figura 2.

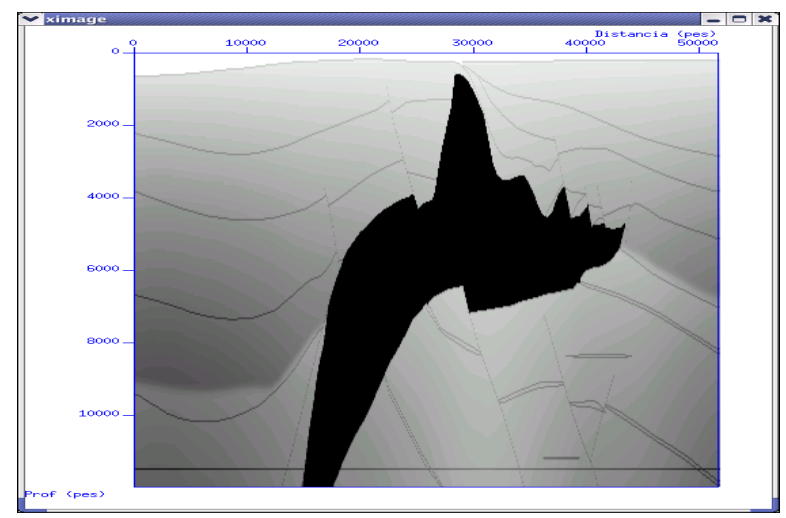

Figura 2 - Campo de velocidades do modelo SEG-EAGE.

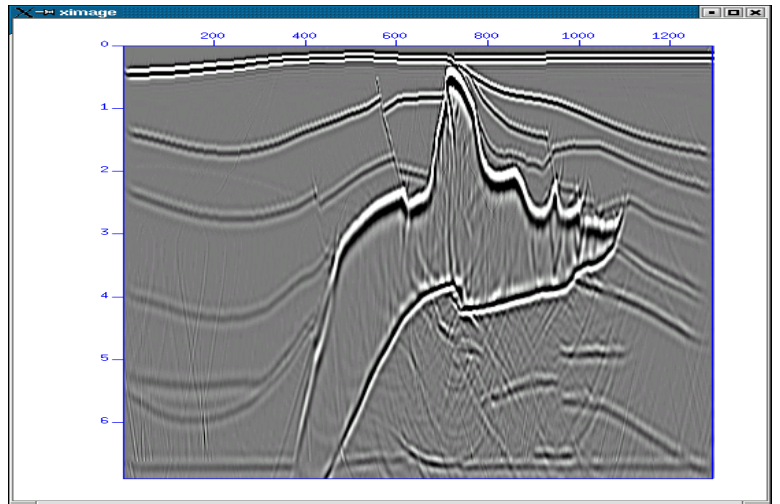

Figura 3 - Resultado da migração com o método Split-Step pós-empilhamento modelo SEG - EAGE.

O dado sintético Marmousi é um dado acústico 2D de estrutura complexa baseado na geologia da bacia de Cuanza em Angola e consiste de 240 famílias com intervalo de amostragem de $4 \mathrm{~ms}$ e com 750 amostras por traço. Seu campo de velocidade é mostrado na Figura 4.

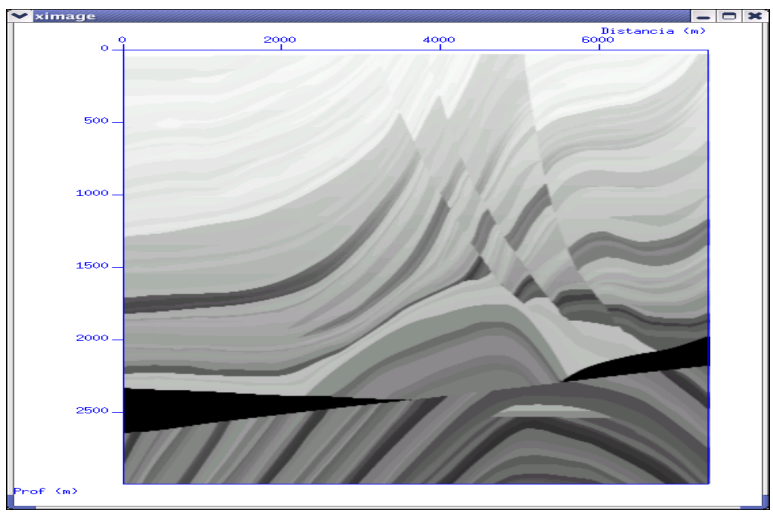

Figura 4 - Campo de velocidades do modelo Marmousi. 


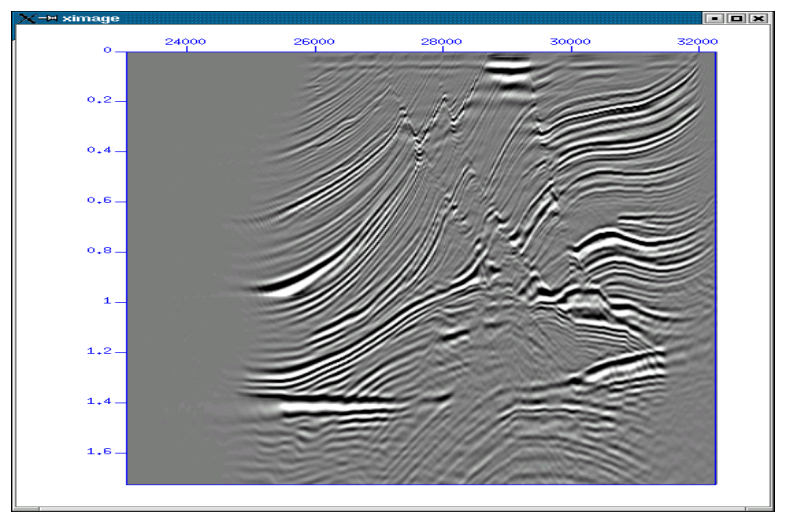

Figura 5 - Resultado da migração com o método Split-Step pré-empilhamento modelo Marmousi

Utilizando o programa desenvolvido no LAGEP Centro de Pesquisa em Geofísica e Geologia - CPGG/UFBA, o Ambiente Gráfico para Imageamento Sísmico - AGIS (Figura 1), obtivemos um tempo de processamento para o dado sintético SEG-EAGE, rodando no INFOCLUSTER ITAUTEC $\AA$ utilizando 1 (um) processador Intel ${ }^{\circledR}$ Xeon ${ }^{\circledR}$, de aproximadamente 4 min 40s, aplicando a técnica SplitStep pós-empilhamento em profundidade. Já o tempo de processamento para o dado sintético Marmousi, rodando no mesmo INFOCLUSTER ITAUTEC $\AA$ e utilizando 32 (trinta e dois) processadores Intel ${ }^{\circledR}$ Xeon $₫$, obtivemos aproximadamente o tempo de 12 min 30s, aplicando-se a técnica de migração Split-Step pré-empilhamento em profundidade.

\section{Discussão e Conclusões}

O sistema operacional Linux tem a fama de ser um sistema complicado, apresentando na maioria das vezes instruções e comandos digitados diretamente no terminal. Assim, a criação e implementação de uma interface gráfica para a execução dos programas de imageamento de dados sísmicos, possibilitarão aos alunos e pesquisadores do LAGEP/CPGG/UFBA um conforto maior na utilização desses programas. Com a criação desse ambiente gráfico pretendemos que os programas de imageamento sísmico possam ser utilizados de forma mais amigável. Além disso, este sistema visa possibilitar a incorporação de novos algoritmos de migração e, por conseguinte, também permitir a realização de testes e a execução dos diversos programas adicionados, de uma forma mais rápida e simples.

Algumas das partes do programa AGIS (Ambiente Gráfico para Imageamento Sísmico) podem ser visto nas Figuras 6, 7, 8 e 9.

\section{Agradecimentos}

Os autores agradecem ao CTPETRO/CNPq-FINEP pelo apoio à rede Cooperativa de Pesquisa em Risco Exploratório, a PETROBRAS, a CAPES e ao CPGG/UFBA pelo apoio no desenvolvimento deste trabalho.

\section{Referências}

BLANCHETTE, J., SUMMERFIELD, M., 2004. C++ GUI Programming with Qt 3. Association with Trolltech Press, $464 \mathrm{p}$.

DALHEIMER, M. K., 1999. Programação em Qt: como desenvolver aplicações GUI portáveis em Unix e Win32. Trad. Alessandra Duarte. Rio de Janeiro: Editora Ciência Moderna Ltda., 335p.

GOTTFRIED, B. S., 1993. Programando em C. Trad. Ana Beatriz Correa da Costa Parra. São Paulo: Makron Books, 574p.

JAMSA, K., 1994. Salvo pelo... C++. Trad. Simone Barbosa de Oliveira. Rio de Janeiro: LTC Livros Técnicos e Científicos Editora, 256p.

PESTANA, R. C., LOGRADO, J. C. G., STOFFA, P. L., 2003. Migração 2-D pré-empilhamento em profundidade de ondas planas utilizando técnicas de correção de fase em duas etapas. VIII Congresso Internacional da Sociedade Brasileira de Geofísica, Rio de Janeiro - RJ.

ALDUNATE, G. C., 2002. Migração Pré-empilhamento Usando Operadores de Extrapolação Split-Step. Dissertação (Mestrado em Geofísica) - Universidade Federal da Bahia, Agência Nacional do Petróleo. Orientador: Reynam da Cruz Pestana. 


\begin{tabular}{|c|c|c|}
\hline$g^{g}-m$ Ambiente Gráfico $p$ & magjeamento Sísmico & $\ln x$ \\
\hline Arquivo Eazer Executa & & \\
\hline $\begin{array}{c}\text { Migração } \\
\text { Pós-empilhamento }\end{array}$ & $\begin{array}{c}\text { Migraçãa } \\
\text { Pré-empilhamento }\end{array}$ & $\begin{array}{l}\text { Migraçäo } \\
\text { Pré-empilhamento } \\
\text { em Paralelo }\end{array}$ \\
\hline$C$ SUMIGSPLIT & $\sim$ SUMIGPREFD & $\sim$ SUMIGPREFD-MPI \\
\hline C SUMIGPSPI & $C$ SUMIGPREFFD & $\sim$ SUMIGPREFFD-MPI \\
\hline$C$ SUMIGFD & $r$ SUMIGPREPSPI & $C$ SUMIGPREPSPI-MPI \\
\hline$C$ SUMIGFFD & C SUMIGPRESP & ( ) SUMIGPRESP-MPI \\
\hline & & $C$ SUMIGPRESSPSPI-MPI \\
\hline $\begin{array}{r}\text { SUMIGPRESP-MPI - The 2-D } \\
\text { migration }\end{array}$ & $\begin{array}{l}\text { ck common-shot split-step } \\
\text { INUX Cluster with MPI }\end{array}$ & \\
\hline $\begin{array}{l}\text { mpirun -np \#np sumigpresp-mp } \\
\text { where } n \text { ntp }=\text { number of nodes }\end{array}$ & ata >outfile [parameters] & \\
\hline 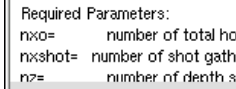 & $\begin{array}{l}\text { aj output samples } \\
\text { be migrated }\end{array}$ & - \\
\hline
\end{tabular}

Figura 6 - AGIS - Tela principal mostrando as opções de métodos para migração usando o pacote SU.

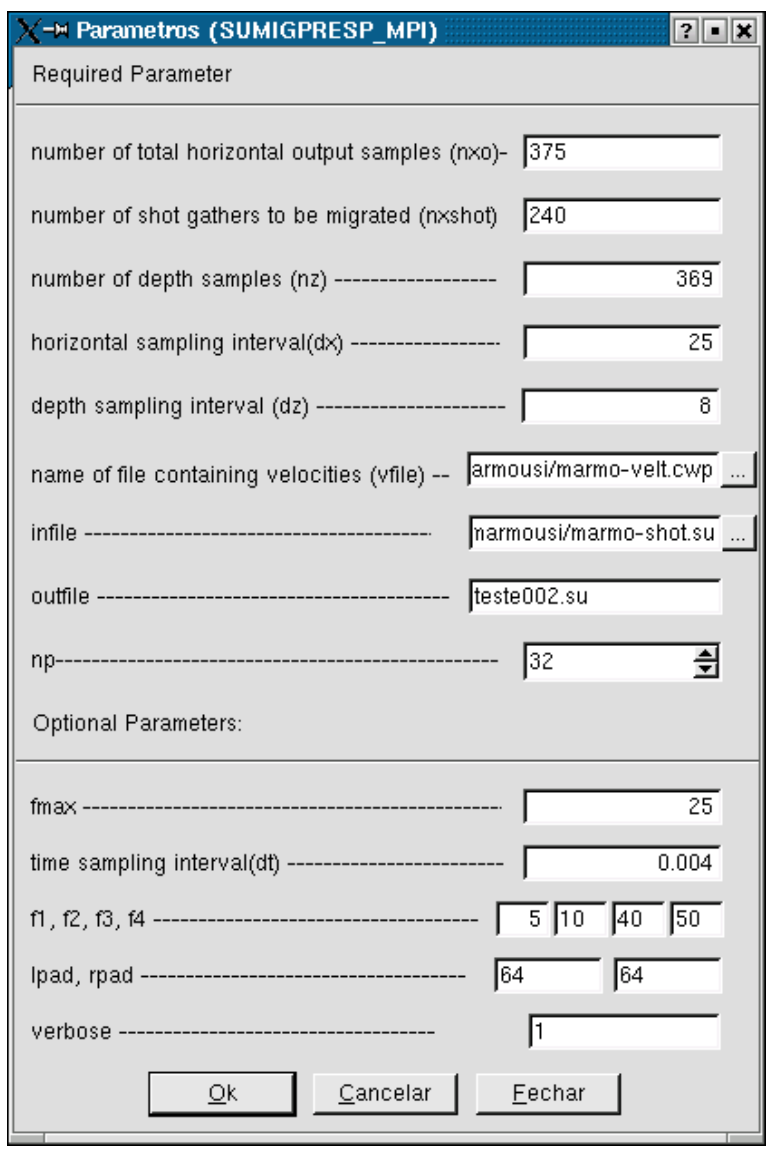

Figura 7 - Tela de parâmetros do método SplitStep pré-empilhamento em paralelo do programa AGIS.

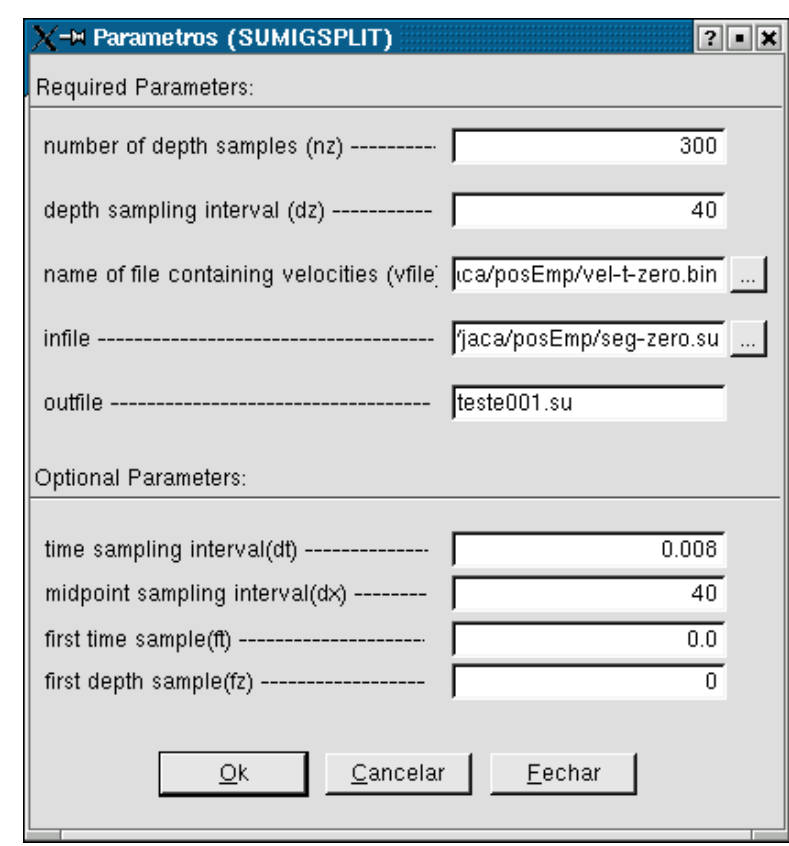

Figura 8 - Tela de parâmetros do método Split-Step pós-empilhamento do programa AGIS.

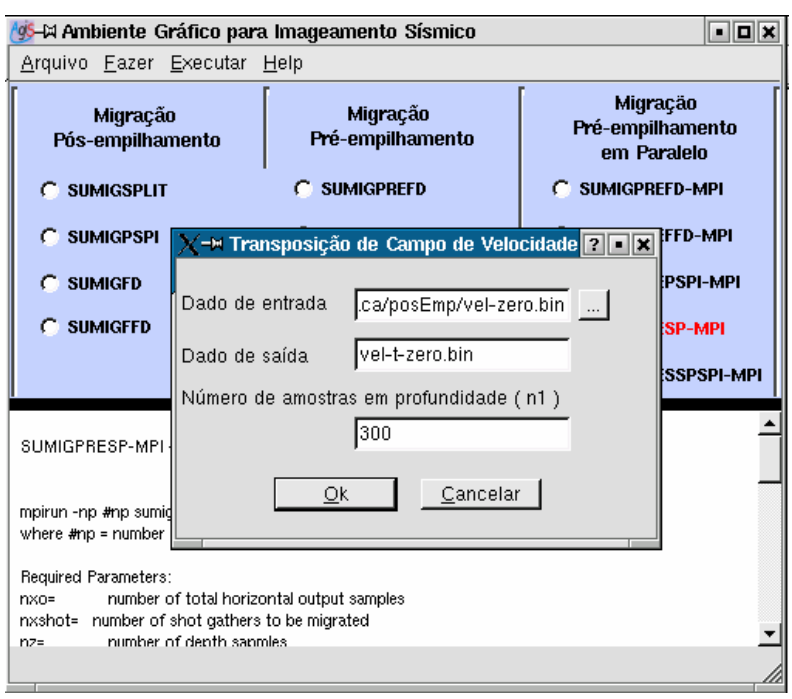

Figura 9 - Fazendo transposição de campo de velocidade com o programa AGIS. 